\title{
A PROBLEM IN COOLING FIN DESIGN
}

\author{
BY
}

\author{
I. E. BOBISUD AND J. F. CALVERT
}

Department of Mathematics, University of Idaho, Moscow, Idaho

\begin{abstract}
We consider two related one-dimensional steady-state problems of heat conduction/radiation in a cooling fin. In both problems the solution consists of the value of a parameter (length of the fin) and a monotone temperature distribution in the fin. Three boundary conditions are accordingly imposed: temperature at both ends of the fin and heat flux at one end. We show that these problems have solutions only for data satisfying certain constraints, and we obtain sufficient conditions for existence. Good estimates for the required fin length are also provided.
\end{abstract}

Introduction and formulation. Consider a cooling fin (or spine, or an array of stacked fins or spines) projecting into the region $0 \leq z \leq a$ from an object whose edge lies at $z=0$. We suppose that the fin is to conduct away from $z=0$ a specified amount of heat per unit time and that the object maintains a temperature $T$ at $z=0$; thus if $U$ denotes the temperature in the fin we have the boundary conditions

$$
\left.\frac{\partial U}{\partial z}\right|_{z=0}=-b,\left.\quad U\right|_{z=0}=T .
$$

For the steady state we have

$$
U^{\prime \prime}(z)-\varphi\left(z, U(z), U^{\prime}(z), a\right)=0,
$$

where $\varphi$ represents heat radiation from the fin; specifically, $\varphi\left(z, U, U^{\prime}, a\right)$ denotes heat radiation per unit length of fin located at position $z \in[0, a]$ when the temperature at $z$ is $U$, the temperature gradient is $U^{\prime}$, and the fin occupies the region $0 \leq z \leq a$. Our goal is to establish the existence of a fin length $a$ such that the temperature at the end $z=a$ of the array is a given value $T_{0}<T$. This third boundary condition may come about either because the fin is to be connected to a heat reservoir at temperature $T_{0}$ or because it is desired to have the end of the fin at a safely cool temperature. (In the latter case we tacitly assume that the rate of heat loss through this end of the fin is small enough to be ignored, except in the last section.) Moreover, we require a fin length for which

Received March 11, 1997.

1991 Mathematics Subject Classification. Primary 34B99.

Key words and phrases. Cooling fins, existence.

E-mail address: bobisud@uidaho.edu

E-mail address: calvert@uidaho.edu 


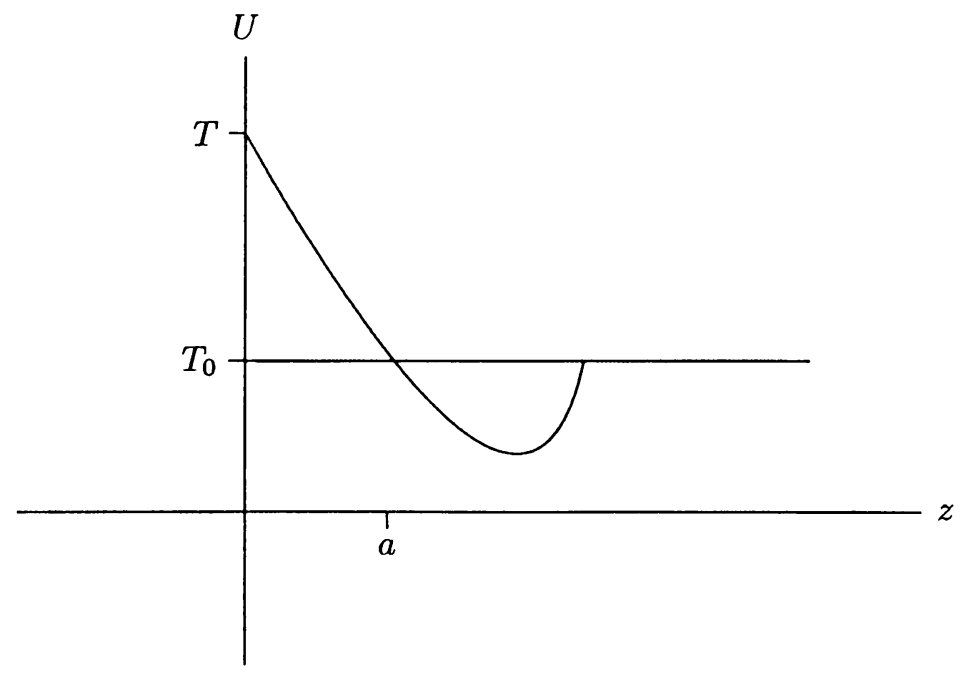

FIG. 1

the temperature distribution $U$ is monotone (if $T_{0}$ exceeds ambient temperature, there may exist a non-monotone solution in a longer fin as well; see Fig. 1). We shall indeed assume that $T_{0}$ exceeds the ambient temperature; thus $T_{0} \leq U(z) \leq T$ must hold.

The change of variable $z=a x, u(x)=U(a x)$ for $0 \leq x \leq 1$ seems natural. With $\psi(x, u, v, a) \equiv a^{2} \varphi(a x, u, v / a, a)$, our problem becomes that of determining a pair $(a, u)$ such that $u$ is monotone and

$$
\begin{gathered}
u^{\prime \prime}(x)=\psi\left(x, u(x), u^{\prime}(x), a\right), \\
u^{\prime}(0)=-a b, \quad u(0)=T, \quad u(1)=T_{0} .
\end{gathered}
$$

To determine a reasonable form to assume for the function $\psi$ as well as to obtain some notion of solvability, we examine a pair of examples. Consider as a first example a single straight fin of uniform thickness $w$ and length $a$, attached to an object of temperature $T$. If the free end of the fin is to have pre-assigned temperature $T_{0}$, we wish to determine the least value of $a$ such that a fin of length $a$ will radiate heat at a given rate. Letting $\theta$ denote temperature above ambient, we have the problem $[1$, p. 86]

$$
\frac{d^{2} \theta}{d z^{2}}=\frac{2 h}{w k} \theta \quad(0 \leq z \leq a), \quad \theta^{\prime}(0)=-b, \quad \theta(0)=T, \quad \theta(a)=T_{0} .
$$

The change of variables suggested above reduces this problem to

$$
u^{\prime \prime}(x)=a^{2} \frac{2 h}{w k} u(x) \quad(0 \leq x \leq 1), \quad u^{\prime}(0)=-a b, \quad u(0)=T, \quad u(a)=T_{0} .
$$

To get a feel for the existence question for such a problem, we examine the explicit solution of this elementary example. For convenience set $\omega^{2}=2 h /(w k)$; then

$$
u(x)=\left(T-\frac{b}{\omega}\right) \cosh a \omega x+\frac{b}{\omega} e^{-a \omega x}
$$


satisfies the differential equation and the boundary conditions at $x=0$. The final boundary condition, $u(1)=T_{0}$, may be viewed as an equation determining the fin length a. Indeed, we have the quadratic

$$
\left(T-\frac{b}{\omega}\right)\left(e^{a \omega}\right)^{2}-2 T_{0}\left(e^{a \omega}\right)+\left(T+\frac{b}{\omega}\right)=0
$$

in $e^{a \omega}$. If $b=\omega T, a$ is the unique value $\left(\ln T-\ln T_{0}\right) / \omega>0$. If $b \neq \omega T$, we have

$$
e^{a \omega}=\frac{T_{0} \pm \sqrt{T_{0}^{2}-T^{2}+b^{2} \omega^{-2}}}{T-b \omega^{-1}} .
$$

Thus in this case there are no real solutions if $T^{2}>T_{0}^{2}+b^{2} \omega^{-2}$ and two real solutions for $e^{a \omega}$ if $T_{0}^{2}+b^{2} \omega^{-2}>T^{2}$. In order that a positive root $a$ exist, it must further be the case that at least the largest value of the right-hand side exceeds one. Suppose first that $T>b \omega^{-1}$ holds. Then the largest root corresponds to the $+\operatorname{sign}$ in (5) and this root exceeds one if and only if

$$
T-T_{0}-b \omega^{-1}<\sqrt{T_{0}^{2}-T^{2}+b^{2} \omega^{-2}}
$$

which on squaring both sides leads directly to the contradiction $T<b \omega^{-1}$ if the lefthand side is positive. On the other hand, if the left side does not exceed zero, we have $T \leq T_{0}+b \omega^{-1}$. This condition plus the condition that $e^{a \omega}$ be real reduces to $b \omega^{-1}<T \leq \sqrt{T_{0}^{2}+b^{2} \omega^{-2}}$.

Suppose next that $T<b \omega^{-1}$; then the largest root for $e^{a \omega}$ must correspond to the minus sign in (5). The condition that this root exceed 1 reduces to $\left(T-b \omega^{-1}\right)\left(T-T_{0}\right)<0$, which is then always valid. In summary, there exists at least one positive solution for a if and only if $T_{0}<T \leq \sqrt{T_{0}^{2}+b^{2} \omega^{-2}}$ holds. Multiple solutions for a may exist, as in Fig. 1.

A similar problem is that of determining the length of a cooling fin when the section of the fin varies. Consider, for example, a single cooling fin of trapezoidal section occupying $0 \leq z \leq a$, as in Fig. 2. Let $w_{1}$ denote the width of the base of the fin and $w_{2}>0$ the width of the "point" $z=a$ of the fin; we suppose that $w_{1}$ and $w_{2}$ are fixed values (alternatively, they could be suitable known functions of the fin length $a$ ). Let the base of the fin have temperature $T$ and the free extremity have temperature $T_{0}$. The problem is

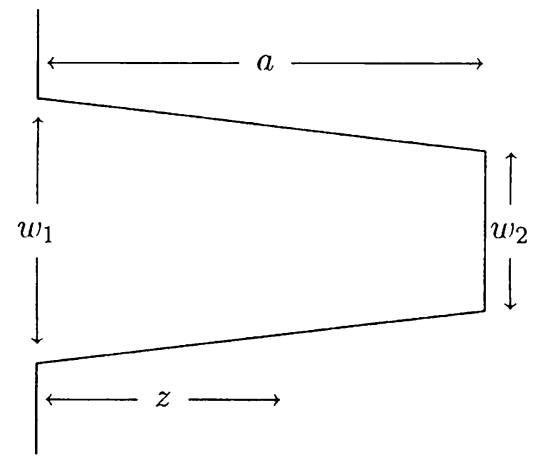

FIG. 2 
to determine the minimum length $a$ such that the fin will radiate a preassigned quantity of heat, i.e., such that $\left.\frac{\partial \theta}{\partial z}\right|_{z=0}$ has a given value. After a change to our variables, the differential equation for the temperature in a trapezoidal fin of fixed length $a$ is given in $[1$, p. 85$]$ as

$$
\theta^{\prime \prime}-\frac{1}{d a-z} \theta^{\prime}-\frac{2 h}{k} \sqrt{\frac{a^{2} d^{2}}{w_{1}^{2}}+\frac{1}{4}} \frac{1}{d a-z} \theta=0
$$

where $d=w_{1} /\left(w_{1}-w_{2}\right)>1$ and $h$ and $k$ are physical constants, the film coefficient and the thermal conductivity, respectively. Our problem is thus to determine a minimal length $a$ such that (6) has a solution satisfying the boundary conditions

$$
\theta^{\prime}(0)=-b, \quad \theta(0)=T, \quad \theta(a)=T_{0}
$$

After the change of variables $z=a x, u(x)=\theta(a x)$, this problem has the form

$$
u^{\prime \prime}(x)=p(x, a) u^{\prime}(x)+a \phi(a) q(x) u(x), \quad 0 \leq x \leq 1
$$

with the boundary conditions (2). Here

$$
p(x, a)=\frac{1}{d-x}, \quad \phi(a)=\frac{2 h}{k} \sqrt{\frac{a^{2} d^{2}}{w_{1}^{2}}+\frac{1}{4}}, \quad q(x)=\frac{1}{d-x} .
$$

Note that $p$ is in fact independent of $a$. Since (7) is more general than (3), our first goal is to obtain conditions guaranteeing that problems of the form (7), (2) have solutions $(a, u(\cdot))$ for monotone $u$. To obtain such conditions that are as weak as possible, we use an abstract fixed-point theorem, the topological transversality theorem of Granas [2, 3].

Of particular interest in applications is a suitable lower bound $A_{l}$ (cf. Theorem 1) on the fin length $a$. For if $A_{l}$ is unsatisfactorily large, then revision of the specifications for the fin is clearly necessary. The upper bound $A_{u}$ on possible fin length may similarly be used to guarantee a reasonable value for $a$ before extensive calculation. We devote some consideration to obtaining a good upper bound under the assumption that a solution does exist. In the final section we consider a "dual" problem in which the temperature at both ends of the fin is prescribed and the free end radiates heat; the length of the fin is again a parameter to be determined.

Existence. To generalise the requirement $T_{0}<T<\sqrt{T_{0}^{2}+b^{2} \omega^{-2}}$ for the problem (4), we want to obtain sufficient conditions for existence of a positive, monotone solution $(a, u(\cdot))$ for the problem

$$
\begin{gathered}
u^{\prime \prime}(x)=p(x, a) u^{\prime}(x)+a \phi(a) q(x) u(x), \quad 0 \leq x \leq 1, \\
u^{\prime}(0)=-a b, \quad u(0)=T, \quad u(1)=T_{0} .
\end{gathered}
$$


This formulation includes the two fin problems considered in the introduction. To ease our notation we define the following constants:

$$
\begin{aligned}
& \Phi=\sup _{a \in(0, \infty)} e^{\int_{0}^{1} p(s, a) d s} /\left(T_{0} \int_{0}^{1} q(t) d t\right), \\
& Q=\left(T^{2}-T_{0}^{2}\right) \sup _{(t, a) \in[0,1] \times(0, \infty)}\left(q(t) e^{-2 \int_{0}^{t} p(s, a)} d s\right), \\
& C=1 / \inf _{a \in(0, \infty)} \int_{0}^{1} e^{\int_{0}^{x} p(s, a) d s} d x \\
& A_{u}=\frac{\Phi Q+\sqrt{\Phi^{2} Q^{2}+4 C^{2}\left(T-T_{0}\right)^{2}}}{2 b} \\
& A_{l}=\left(T-T_{0}\right) /\left(b \sup _{a \in(0, \infty)} \int_{0}^{1} e^{\int_{0}^{x} p(s . a) d s} d x\right) .
\end{aligned}
$$

We use the topological transversality theorem of Granas $[2,3]$ to establish the following result:

Theorem 1. Let $0<T_{0}<T$. Assume that $\phi>0$ and that $y \phi(y)$ is nondecreasing on $(0, \infty)$, that $q>0, q \in C[0,1]$, and that $p \geq 0$ is continuous in $(x, a) \in[0,1] \times(0, \infty)$ with $\int_{0}^{1} p(x, a) d x$ bounded for $a \in(0, \infty)$. Then if (8)-(9) has a solution $(a, u)$ with $a>0$ and $u>0$ nonincreasing, it must be that $A_{l} \leq a<A_{u}$. Suppose in addition that

$$
\frac{T-T_{0}}{T}>A_{u} \phi\left(A_{u}\right) \max _{[0,1]} q(x) \sup _{(0, \infty)}\left(\int_{0}^{1} e^{\int_{0}^{t} p(s, a) d s} d t \int_{0}^{1} e^{-\int_{0}^{t} p(s, a) d s} d t\right) .
$$

Then (8)-(9) possesses a solution $(a, u)$ with $a>0$ and $u>0$ decreasing.

Proof. As in any degree-theoretic argument, we need to consider a suitable oneparameter family of problems in which (8)-(9) is embedded. We use (9) and

$$
u^{\prime \prime}(x)=p(x, a) u^{\prime}(x)+\lambda a \phi(a) q(x) u(x), \quad 0 \leq x \leq 1
$$

where $\lambda \in[0,1]$ is the parameter and we suppress the dependence of $u$ and $a$ on $\lambda$ in the notation. If $u$ is a monotone solution of the problem (11), (9) for some $a>0$, then we have $T_{0} \leq u(x) \leq T$, yielding the desired a priori bounds on $u$, and $u^{\prime}(x) \leq 0$. Use of the obvious integrating factor allows (11) to be written as

$$
\left(e^{-\int_{0}^{x} p(s, a) d s} u^{\prime}(x)\right)^{\prime}=\lambda a \phi(a) e^{-\int_{0}^{x} p(s, a) d s} q(x) u(x) .
$$

Integration over $[0,1]$ and use of $u^{\prime} \leq 0$ yields

$$
\begin{aligned}
b & \geq \lambda \phi(a) \int_{0}^{1} e^{-\int_{0}^{t} p(s, a) d s} q(t) u(t) d t \\
& \geq \lambda \phi(a) \cdot T_{0} e^{-\int_{0}^{1} p(s, a) d s} \int_{0}^{1} q(t) d t
\end{aligned}
$$


this provides an upper bound on $\lambda \phi(a)$ which we may write as $\lambda \phi(a) \leq b \Phi$. Rewriting (12) in the form

$$
\begin{aligned}
& \left(e^{-\int_{0}^{x} p(s . a) d s} u^{\prime}(x)\right)\left(e^{-\int_{10}^{x} p(s . a) d s} u^{\prime}(x)\right)^{\prime} \\
& \quad=\lambda a \phi(a) e^{-2 \int_{0}^{x} p(s . a) d s} q(x) u(x) u^{\prime}(x)
\end{aligned}
$$

and integrating over $[0, x] \subset[0,1]$ yields

$$
\left(e^{-\int_{0}^{x} p(s . a) d s} u^{\prime}(x)\right)^{2}-a^{2} b^{2}=2 \lambda a \phi(a) \int_{0}^{x} e^{-2 \int_{0}^{t} p(s . a) d s} q(t) u(t) u^{\prime}(t) d t .
$$

From this we get (again because $u^{\prime} \leq 0$ ) that

$$
\left(e^{-\int_{0}^{r} p(s . a) d s} u^{\prime}(x)\right)^{2} \geq a^{2} b^{2}-\Phi Q a b .
$$

If the right-hand side of (14) is nonpositive, then

$$
a \leq \frac{1}{b} \Phi Q
$$

if the right-hand side of (14) is positive, then

$$
u^{\prime}(x) \leq-\sqrt{a^{2} b^{2}-\Phi Q a b} e^{\int_{0}^{x} p(s . a) d s} .
$$

Integrating this latter inequality over $[0,1]$ gives us that

$$
T-T_{0} \geq \int_{0}^{1} e^{\int_{0}^{x} p(s . a) d s} d x \sqrt{a^{2} b^{2}-\Phi Q a b} \geq \frac{1}{C} \sqrt{a^{2} b^{2}-\Phi Q a b} ;
$$

this is possible only if

$$
a \leq \frac{\Phi Q+\sqrt{\Phi^{2} Q^{2}+4 C^{2}\left(T-T_{0}\right)^{2}}}{2 b} \equiv A_{u} .
$$

Noting that the right-hand side of (15) exceeds $\Phi Q / b$, we see that $A_{u}$ always yields an upper bound on $a$.

In view of the obvious physical requirement $a>0$, we need to obtain a positive lower bound on $a$. From the nonnegativity of the right-hand side of (12) and from the boundary conditions (9) we get on integrating over $[0, x]$ that

$$
u^{\prime}(x) \geq-a b e^{\int_{0}^{x} p(s . a) d s}
$$

a subsequent integration over $[0,1]$ yields

$$
T-T_{0} \leq a b \sup _{a \in(0 . x)}\left(\int_{0}^{1} e^{\int_{0}^{x} p(s . a) d s} d x\right)
$$

which provides the required lower bound on $a: a \geq A_{l}$.

From (12) and the bounds already established we see that there is a constant $k$ (independent of $a$ and $\lambda \in[0,1])$ such that

$$
\left|\left(e^{-\int_{0}^{*} p(s . a) d s} u^{\prime}(x)\right)^{\prime}\right| \leq k .
$$

An integration over $[0, x]$ gets us that

$$
0 \geq u^{\prime}(x) \geq-(k+a b) \sup _{a \in(0 . x)} e^{\int_{0}^{1} p(s . a) d s},
$$

providing an a priori lower bound since $a$ has been shown to be bounded. The existence of a suitable a priori bound on $u^{\prime \prime}$ now follows from the differential equation (11) itself. 
We now convert the problem (11), (9) to an integral equation formulation. From (12) we have that

$$
u^{\prime}(x)=-a b e^{\int_{0}^{x} p(s, a) d s}+\lambda a \phi(a) \int_{0}^{x} e^{\int_{t}^{x} p(s, a) d s} q(t) u(t) d t
$$

integration over $[0, x]$ now yields

$$
u(x)=T-a b \int_{0}^{x} e^{\int_{0}^{z} p(s, a) d s} d z+\lambda a \phi(a) \int_{0}^{x} \int_{0}^{z} e^{\int_{t}^{z} p(s, a) d s} q(t) u(t) d t d z .
$$

In addition, the boundary condition at $x=1$ implies that

$$
T-T_{0}=a b \int_{0}^{1} e^{\int_{0}^{z} p(s, a) d s} d z-\lambda a \phi(a) \int_{0}^{1} \int_{0}^{z} e^{\int_{t}^{z} p(s, a) d s} q(t) u(t) d t d z .
$$

Suppose conversely that the pair $(a, u)$ with $u \in C[0,1]$ satisfies (17), (18). In order we deduce that $u(1)=T_{0}, u(0)=T, u \in C^{1}, u^{\prime}$ satisfies $(16), u^{\prime}(0)=-a b, u^{\prime} \in C^{1}[0,1]$, and the differential equation (11) is satisfied. Thus $(17),(18)$ is equivalent to (11), (9).

We introduce an operator $\Theta_{\lambda}:(\alpha, v(\cdot)) \mapsto(\beta, w(\cdot))$ by

$$
\begin{gathered}
w(x)=T-\beta b \int_{0}^{x} e^{\int_{0}^{z} p(s, \alpha) d s} d z+\lambda \alpha \phi(\alpha) \int_{0}^{x} \int_{0}^{z} e^{\int_{t}^{z} p(s . \alpha) d s} q(t) v(t) d t d z \\
\beta=\left(T-T_{0}+\lambda \alpha \phi(\alpha) \int_{0}^{1} \int_{0}^{z} e^{\int_{t}^{z} p(s, \alpha) d s} q(t) v(t) d t d z\right) /(b P(\alpha))
\end{gathered}
$$

where

$$
P(\alpha) \equiv \int_{0}^{1} e^{\int_{0}^{z} p(s, \alpha) d s} d z
$$

Clearly, fixed points of the operator $\Theta_{\lambda}$ are solutions of $(17),(18)$, and conversely. Let $\|\cdot\|_{0}$ be the supremum norm on $[0,1]$ and let $\|y\|=\max \left(\|y\|_{0},\left\|y^{\prime}\right\|_{0},\left\|y^{\prime \prime}\right\|_{0}\right)$ for $y \in$ $C^{2}[0,1] ; \mathcal{B}=(R,|\cdot|) \times\left(C^{2}[0,1],\|\cdot\|\right)$, where $R$ is the real line, is a Banach space. Then

$$
K=\left\{(a, y) \in \mathcal{B}: a \geq 0 \text { and } y^{\prime} \leq 0\right\}
$$

is a convex subset of $\mathcal{B}$, and

$$
U=\left\{(a, y) \in K: A_{l}-\varepsilon<a<A_{u}+\varepsilon, T_{0}-\varepsilon<y<T+\varepsilon,-k_{1}-\varepsilon<y^{\prime},\left\|y^{\prime \prime}\right\|_{0}<k_{2}+\varepsilon\right\}
$$

is an open subset of $K$; here $\varepsilon$ is arbitrary in the interval $\left(0, \max \left(A_{l}, T_{0}\right)\right)$ and $-k_{1} \leq$ $u^{\prime} \leq 0,\left\|u^{\prime \prime}\right\|_{0} \leq k_{2}$ are the already established bounds on the derivatives of monotone solutions of (11), (9). Our a priori estimates show that no fixed point of $\Theta_{\lambda}$ for any $\lambda \in[0,1]$ lies on the boundary of $U$.

We need to show that $\Theta$. is a compact map of $[0,1] \times \bar{U}$ into $\mathcal{B}$. Clearly, $\Theta$. is continuous. Let then $\lambda \in[0,1], 0<A_{l}-\varepsilon \leq \alpha \leq A_{u}+\varepsilon$, and let $v(x)$ satisfy $0<T_{0}-\varepsilon \leq v \leq T+\varepsilon$, $-k_{1}-\varepsilon \leq v^{\prime} \leq 0,\left\|v^{\prime \prime}\right\|_{0} \leq k_{2}+\varepsilon$ so that $(\alpha, v) \in \bar{U}$. Let $(\beta, w(\cdot))$ be the image under $\Theta$. of $(\lambda,(\alpha, v))$. From the boundedness of $\alpha$ we see from (20) that possible values of $\beta$ are bounded above and that $\beta>0$; so $\beta$ lies in a fixed compact set. From (19) we see that the set of all values of $w$ is uniformly bounded; from (19) we also have that

$$
w^{\prime}(x)=-\beta b e^{\int_{0}^{x} p(s, \alpha) d s}+\lambda \alpha \phi(\alpha) \int_{0}^{x} e^{\int_{t}^{x} p(s, \alpha) d s} q(t) v(t) d t
$$


and then that

$$
w^{\prime \prime}(x)=p(x, \alpha) w^{\prime}(x)+\lambda \alpha \phi(\alpha) q(x) v(x) .
$$

From (21) and the boundedness of $\alpha, \beta$, and $v$ we conclude that $\left\{w^{\prime}\right\}$ is uniformly bounded; consequently; $\{w\}$ is equicontinuous in the norm $\|\cdot\|_{0}$. From (22) we get accordingly that $\left\{w^{\prime \prime}\right\}$ is uniformly bounded, and hence $\left\{w^{\prime}\right\}$ is equicontinuous in the $\|\cdot\|_{0}$-norm. Now (22) yields directly the equicontinuity of $\left\{w^{\prime \prime}\right\}$ in the norm $\|\cdot\|_{0}$. We conclude that the mapping $(\lambda,(\alpha, v)) \mapsto w$ satisfies the hypotheses of the Ascoli-Arzela Lemma and so is compact; the boundedness of $\{\beta\}$ then guarantees that $\Theta$. is compact on $[0,1] \times \bar{U}$.

There remains principally to show that the image of $\Theta$. lies in $K$; i.e., in the notation of the previous paragraph, that $\beta>0$ and $w^{\prime} \leq 0$. The first is clear from (20) since $T_{0}<T$. From (21) $w^{\prime} \leq 0$ will be a consequence of

$$
b \beta \geq \alpha \phi(\alpha) \int_{0}^{1} e^{-\int_{0}^{t} p(s, \alpha) d s} q(t) v(t) d t
$$

for all $(\alpha, v) \in \bar{U}$. From (20) we get that $b \beta>\left(T-T_{0}\right) / P(\alpha)$; since $v(t) \leq T+\varepsilon,(23)$ will follow for sufficiently small positive $\varepsilon$ (which we are still free to pick) if

$$
\frac{T-T_{0}}{T}>\alpha \phi(\alpha) P(\alpha) \int_{0}^{1} e^{-\int_{0}^{t} p(s, \alpha) d s} q(t) d t .
$$

From the definition of $P(\alpha)$ this will be the case if the inequality (10) in the statement of Theorem 1 holds.

If $\Theta_{0}$ is an essential map, then by the topological transversality theorem $[2,3]$ so is $\Theta_{1}$, whence $\Theta_{1}$ has a fixed point. This fixed point is the desired solution of (11), (9) for $\lambda=1$, and we are done. Unfortunately, $\Theta_{0}$ is not a constant map since the image of $(\alpha, v)$ depends on $\alpha$ (though not on $v$ ). We now show that $\Theta_{0}$ is nevertheless essential. To this end we consider the map $\Psi_{\mu}:(\alpha, y(\cdot)) \mapsto(\beta, z(\cdot))$ defined by

$$
\begin{gathered}
z(x)=T-\beta b \int_{0}^{x} e^{\int_{0}^{t} p(s \cdot \mu \alpha+(1-\mu) \hat{\alpha}) d s} d t, \\
\beta=\frac{T-T_{0}}{b \int_{0}^{1} e^{\int_{0}^{t} p(s \cdot \mu \alpha+(1-\mu) \hat{\alpha}) d s} d t}
\end{gathered}
$$

for $\mu \in[0,1]$, where $\hat{\alpha}$ is some fixed value in $\left(A_{l}, A_{u}\right)$. Clearly $\Psi_{1}=\Theta_{0}$, and $\Psi_{0}$ is a constant map; it is a straightforward exercise to check that $\Psi$. : $[0,1] \times \bar{U} \rightarrow K$ is a compact homotopy. If $(\alpha, y)$ is a fixed point of $\Psi_{\mu}$ for $\mu \in[0,1]$, from (25) we have that

$$
A_{u} \geq \frac{T-T_{0}}{b \inf _{\alpha \in(0 . x)} \int_{0}^{1} e^{\int_{0}^{t} p(s . \alpha) d s} d t} \geq \alpha \geq \frac{T-T_{0}}{b \sup _{\alpha \in(0 . x)} \int_{0}^{1} e^{\int_{0}^{t} p(s . \alpha) d s} d t}=A_{l},
$$

the first inequality following from $A_{u} \geq C\left(T-T_{0}\right) / b$, itself a consequence of (15). The same a priori bounds on solutions of (11), (9) continue to hold because $z$ satisfies the problem

$$
z^{\prime \prime}(x)=p(x, \mu \alpha+(1-\mu) \hat{\alpha}) z^{\prime}(x), \quad z(0)=T, \quad z(1)=T_{0}, \quad z^{\prime}(0)=-\alpha b
$$


and $A_{l} \leq \mu \alpha+(1-\mu) \hat{\alpha} \leq A_{u}$ since $\alpha$ and $\hat{\alpha}$ satisfy these inequalities. Thus the compact homotopy $\Psi$. is fixed-point free on $\partial U$, whence by the topological transversality theorem $\Psi_{1}=\Theta_{0}$ is essential because the constant map $\Psi_{0}$ is.

We remark that the restriction $p \geq 0$ is easily removed provided we require that

$$
\sup _{x \in[0.1] . a \in(0 . \infty)} \int_{0}^{x} p(s, a) d s<\infty .
$$

For the first example of the introduction the right-hand side of the inequality (10) can be calculated easily; we find that

$$
\frac{1}{T}>\frac{T-T_{0}}{4 b^{2}}\left[\frac{T+T_{0}}{T_{0}}+\sqrt{\left(\frac{T+T_{0}}{T_{0}}\right)^{2}+4}\right]^{2} \omega^{2} .
$$

This agrees qualitatively with the result obtained from the explicit solution: the problem is solvable for $T$ greater than, and sufficiently close to, $T_{0}$. To get a quantitative feel for the severity of the condition (10), we consider a numerical example. Using $k=132$ $\mathrm{Btu} / \mathrm{hr}-\mathrm{ft}-{ }^{\circ} \mathrm{F}, h=1.6 \mathrm{Btu} / \mathrm{hr}-\mathrm{ft}^{2}{ }^{\circ}{ }^{\circ} \mathrm{F}$ (these values for aluminum are from Chapman [1, pp. 71-72]) and a fin thickness $w$ of $1 / 8 \mathrm{in}$, we find $\omega \sim 1.53$. If the fin is to radiate, say, $400 \mathrm{Btu} / \mathrm{hr}$ per foot of fin width, then $b \sim 291$. Suppose the fin is to be $T_{0}=50^{\circ} \mathrm{F}$ above ambient temperature at the free end $z=a$. Then the exact condition obtained in the introduction implies that a suitable fin exists provided that the temperature $T$ at the base of the fin lies between $50^{\circ} \mathrm{F}$ and $196.6^{\circ} \mathrm{F}$ above ambient temperature, while the sufficient condition just obtained from the theorem reduces to $50^{\circ}<T<90.6^{\circ}$ above ambient. For $T=80^{\circ}$ the estimate $A_{l} \leq a \leq A_{u}$ for the fin length becomes $.103 \leq a \leq .303$. The actual value of $a$ is found to be .106 , approximately.

Improved upper bound on fin length. The numerical example above shows that the $a$ priori bounds on fin length $a$ obtained in the preceding section are not particularly close, especially the upper bound $A_{u}$. Note that in obtaining a new bound we may make free use of the bounds $A_{l} \leq a \leq A_{u}$ of Theorem 1. In particular, these bounds may themselves be used in improving the bounds of that theorem: e.g., we may replace $\sup _{0<a<\infty}$ with $\sup _{A_{l} \leq a \leq A_{u}}$ to obtain new and stronger estimates $A_{l}^{\prime} \leq a \leq A_{u}^{\prime}$. An instance of this occurs in the theorem below: in the second example of the introduction $\phi(a) / a$ is not bounded for $a>0$, but $\max _{a \in\left[A_{l}, A_{u}\right]} \phi(a) / a$ is finite.

THEOREM 2. Let the conditions of Theorem 1, with the exception of (10), hold. Let

$$
\Psi=\sup _{0<x<1, A_{l} \leq a \leq A_{u}}\left(e^{-2 \int_{0}^{x} p(s, a) d s} q(x)\right), \quad \Omega=\inf _{A_{l} \leq a \leq A_{u}} \int_{0}^{1} e^{\int_{0}^{x} p(s . a) d s} d x
$$

and suppose that

$$
b^{2}>\Psi\left(\sup _{A_{l} \leq a \leq A_{u}} \frac{\phi(a)}{a}\right)\left(T^{2}-T_{0}^{2}\right)
$$


Then if there is a solution $(a, u(x))$ of $(2),(7)$ with $u^{\prime}(x) \leq 0, a$ must satisfy the inequality $a \leq \widetilde{A}_{u}$ where $\widetilde{A}_{u}$ is the largest root of

$$
z^{2} b^{2}-z \phi(z) \Psi\left(T^{2}-T_{0}^{2}\right) \leq\left(\frac{T-T_{0}}{\Omega}\right)^{2} .
$$

Suppose also that

$$
\begin{aligned}
\frac{T-T_{0}}{T}> & \widetilde{A}_{u} \phi\left(\widetilde{A}_{u}\right) \max _{x \in[0.1]} q(x) \\
& \times \max _{a \in\left[A_{l}, \tilde{A}_{u}\right]}\left(\int_{0}^{1} e^{\int_{0}^{t} p(s, a) d s} d t \int_{0}^{1} e^{-\int_{0}^{t} p(s, a) d s} d t\right) .
\end{aligned}
$$

Then (8)-(9) has a solution $(a, u)$ with $a>0$ and $u>0$ decreasing.

Proof. The argument is a variant of the one used earlier. From the inequality (14) and the nonpositivity of $u^{\prime}$ we get for $\lambda \in[0,1]$ that

$$
\begin{aligned}
\left(e^{-\int_{0}^{x} p(s, a) d s} u^{\prime}(x)\right)^{2} & \geq a^{2} b^{2}-a \phi(a) \Psi\left(T^{2}-u(x)^{2}\right) \\
& \geq a^{2} b^{2}-a \phi(a) \Psi\left(T^{2}-T_{0}^{2}\right)
\end{aligned}
$$

the last expression is positive by hypothesis. Therefore

$$
u^{\prime}(x) \leq-\sqrt{a^{2} b^{2}-a \phi(a) \Psi\left(T^{2}-T_{0}^{2}\right)} e^{\int_{0}^{x} p(s, a) d s} .
$$

A further investigation yields the inequality of the theorem, which sets a new a priori upper bound on $a$.

The remainder of the argument of Theorem 1 goes through unchanged (except for using $\widetilde{A}_{u}$ instead of $A_{u}$ and $\Omega$ instead of $\left.1 / C\right)$ once we observe that $\widetilde{A}_{u} \geq\left(T-T_{0}\right) /(\Omega b)$, which is needed toward the end of the proof. But since $\widetilde{A}_{u}$ makes an equality of (27), this is immediate.

The hypothesis (26) of Theorem 2 seems to be easy to satisfy in practice, and the upper bound of Theorem 2 is generally much better than that of Theorem 1. Returning to the numerical example considered at the end of the preceding section, we compute the upper bound 0.109 for $a$, a very considerable improvement over the bound 0.303 from Theorem 1. Moreover, the range of temperatures $T$ over which a solution is guaranteed to exist now becomes $50^{\circ}<T<152.1^{\circ}$.

A related problem. Here we consider a cooling fin with prescribed temperature $T$ at the edge $z=0$ of the object to be cooled, where we now suppose that the free end at $z=a$ radiates according to Newton's law of cooling into surroundings at $0^{\circ}$. Thus the boundary condition at $a$ is $d \theta(a) / d z+\left(h_{e} / k\right) \theta(a)=0$, where $h_{e}$ is the film coefficient at the end of the fin and may differ from that of the lateral side of the fin. The fin length $a$ is to be determined by the auxiliary boundary condition that the temperature at the free end is to be $T_{0}<T$. After introducing the variables $x=z / a, u(x)=\theta(a x)$, we have the problem

$$
\begin{gathered}
u^{\prime \prime}(x)=p(x, a) u^{\prime}(x)+\lambda a \phi(a) q(x) u(x) \quad(0<x<1) \\
u(0)=T, \quad u(1)=T_{0}, \quad u^{\prime}(1)=-B a
\end{gathered}
$$


for $\lambda=1$, where we set $B=h_{e} T_{0} / k$ and $p, q, \phi$ are as before. Note that this problem does not reduce to the earlier one under the change of independent variable $x \rightarrow 1-x$.

TheOREm 3. Assume that $0<T_{0}<T$, that $\phi>0$ and $y \phi(y)$ is nondecreasing on $(0, \infty)$, that $q \in C[0,1]$ is positive, and that $p \geq 0$ is continuous in $(x, a) \in[0,1] \times(0, \infty)$ with $\int_{0}^{1} p(x, a) d x$ bounded for $a \in(0, \infty)$. Suppose also that

$$
\frac{T-T_{0}}{T}>A_{u} \phi\left(A_{u}\right) \max _{0 \leq a \leq A_{u}} \int_{0}^{1} \int_{z}^{1} e^{\int_{t}^{z} p(s, a) d s} q(t) d t d z
$$

where

$$
A_{u}=\left(T-T_{0}\right) /\left(B \inf _{0<a<\infty} e^{-\int_{0}^{1} p(s, a) d s}\right) .
$$

Then (29)-(30) with $\lambda=1$ has a solution $(a, u)$ with $a>0$ and $u(x)>0$ nondecreasing.

Proof. We have $0<T_{0} \leq u(x) \leq T$ and $u^{\prime}(x) \leq 0$. An upper bound on $a$ valid for all $\lambda \in[0,1]$ is easily obtained by integrating the inequality

$$
\left(u^{\prime \prime}(x)-p(x, a) u^{\prime}(x)\right) e^{\int_{x}^{1} p(s, a) d s} \geq 0
$$

over $(x, 1)$ to yield

$$
u^{\prime}(x) \leq-B a e^{-\int_{x}^{1} p(s, a) d s} \leq-B a \inf _{a \in(0, \infty)} e^{-\int_{0}^{1} p(s, a) d s} .
$$

A subsequent integration over $(0,1)$ yields the bound $a \leq A_{u}$. From the inequality

$$
\left(e^{\int_{x}^{1} p(s . a) d s} u^{\prime}(x)\right)^{\prime} \leq a \phi(a) e^{\int_{. x}^{1} p(s, a) d s} q(t) u(t) d t,
$$

we get on integrating over $(x, 1)$ that

$$
-u^{\prime}(x) \leq B a+a \phi(a) T \int_{x}^{1} e^{-\int_{x}^{t} p(s, a) d s} q(t) d t
$$

a subsequent integration over $[0,1]$ yields

$$
T-T_{0} \leq B a+T M a \phi(a)
$$

where

$$
M \equiv \max _{0 \leq a \leq A_{u}} \int_{0}^{1} \int_{x}^{1} e^{-\int_{x}^{t} p(s, a) d s} q(t) d t d x
$$

thus setting a lower bound $A_{l}$ on $a$. Suitable a priori bounds on $u^{\prime}$ and $u^{\prime \prime}$ follow much as in the proof of Theorem 1.

As in earlier work, we define an operator $\Theta_{\lambda}:(\alpha, v(\cdot)) \mapsto(\beta, w(\cdot))$ by

$$
\begin{aligned}
w(x)= & T_{0}+B \beta \int_{x}^{1} e^{-\int_{t}^{1} p(s, \alpha) d s} d t \\
& +\lambda \alpha \phi(\alpha) \int_{x}^{1} \int_{z}^{1} e^{\int_{t}^{z} p(s, \alpha) d s} q(t) u(t) d t d z \\
\beta= & \left(T-T_{0}-\lambda \alpha \phi(\alpha) \int_{0}^{1} \int_{z}^{1} e^{\int_{t}^{z} p(s, \alpha) d s} q(t) u(t) d t d z\right) /(B P(\alpha)),
\end{aligned}
$$


where now

$$
P(\alpha) \equiv \int_{0}^{1} e^{-\int_{t}^{1} p(s . \alpha) d s} d t
$$

Then fixed points of $\Theta_{\lambda}$ are precisely the solutions of (29)-(30). $K$ and $U$ are defined as before. Hypothesis (31) guarantees that $\beta>0$. The remaining argument is very like that of Theorem 1, except that now $w^{\prime}(x)<0$ is immediate upon differentiating (34). Details are omitted.

Much improved upper bounds for the length $a$ of the fin can be obtained when $\lambda=1$. For example, on multiplying (33), which is an equality for $\lambda=1$, by $\exp \left(\int_{x}^{1} p(s, a) d s\right) u^{\prime}(x)$ and integrating over $(x, 1)$ we get that

$$
P^{2} u^{\prime}(x)^{2} \geq a^{2} B^{2}+a \phi(a) Q\left(u(x)^{2}-T_{0}^{2}\right),
$$

where we set

$$
P=\max _{a \in\left[A_{l} . A_{u}\right]} e^{\int_{0}^{1} p(s . a) d s}, \quad Q=\min _{x \in[0.1]} q(x)
$$

Further setting

$$
W=\min _{a \in\left[A_{l} . A_{u}\right]} \sqrt{\frac{a}{\phi(a) Q}} B
$$

and recalling that $u^{\prime} \leq 0$, we get that

$$
u^{\prime}(x) \leq-\frac{1}{P} \sqrt{a \phi(a) Q} \sqrt{W^{2}-T_{0}^{2}+u(x)^{2}} .
$$

Division by $\sqrt{u^{2}+W^{2}-T_{0}^{2}}$ and integration over $[0,1]$ yields

$$
\ln \left(\frac{T+\sqrt{T^{2}+W^{2}-T_{0}^{2}}}{T_{0}+W}\right) \geq \frac{1}{P} \sqrt{a \phi(a) Q},
$$

implicitly defining an improved upper bound on $a$.

\section{REFERENCES}

[1] A. J. Chapman, Heat Transfer, 2nd ed., Macmillan, New York, 1967

[2] A. Granas, R. B. Guenther, and J. W. Lee, Nonlinear boundary value problems for ordinary differential equations, Dissertationes Mathematicae 244, 1-128 (1985)

[3] A. Granas, R. B. Guenther, and J. W. Lee, Some general existence principles in the Carathéodory theory of nonlinear differential systems, J. Math. Pures et Appl. 70, 153-196 (1991) 